\title{
Can modified gravity models reconcile the tension between the CMB anisotropy and lensing maps in Planck-like observations?
}

\author{
Bin $\mathrm{Hu}^{1}$ and Marco Raveri ${ }^{2,3}$ \\ ${ }^{1}$ Institute Lorentz, Leiden University, PO Box 9506, Leiden 2300 RA, Netherlands \\ ${ }^{2}$ SISSA - International School for Advanced Studies, Via Bonomea 265, 34136 Trieste, Italy \\ ${ }^{3}$ INFN, Sezione di Trieste, Via Valerio 2, I-34127 Trieste, Italy
}

(Received 27 February 2015; published 11 June 2015)

\begin{abstract}
Planck-2015 data seem to favor a large value of the lensing amplitude parameter, $A_{\mathrm{L}}=1.22 \pm 0.10$, in CMB spectra. This result is in $2 \sigma$ tension with the lensing reconstruction result, $A_{\mathrm{L}}^{\phi \phi}=0.95 \pm 0.04$. In this paper, we simulate several CMB anisotropy and CMB lensing spectra based on Planck-2015 best-fit cosmological parameter values and Planck bluebook beam and noise specifications. We analyze several modified gravity models within the effective field theory framework against these simulations and find that models whose effective Newton constant is enhanced can modulate the CMB anisotropy spectra in a way similar to that of the $A_{\mathrm{L}}$ parameter. However, in order to lens the CMB anisotropies sufficiently, like in the Planck-2015 results, the growth of matter perturbations is substantially enhanced and gives a high $\sigma_{8}$ value. This in turn proves to be problematic when combining CMB anisotropy data from Planck with other data, such as weak lensing from CFHTLenS which favor a smaller amplitude of matter fluctuations.
\end{abstract}

DOI: 10.1103/PhysRevD.91.123515

PACS numbers: 98.80.-k

\section{INTRODUCTION}

Based on the full-mission Planck observations of temperature and polarization anisotropies of the cosmic microwave background (CMB) radiation, Planck-2015 results show that the temperature and polarization power spectra are consistent with the standard spatially flat six-parameter vanilla $\Lambda \mathrm{CDM}$ cosmology with a primordial power-law spectrum of adiabatic scalar perturbations. Hereafter, we call this the base- $\Lambda$ CDM model. On the other hand, the same data, especially the temperature-temperature (TT) spectrum, reveals some tension with the $\mathrm{CMB}$ lensing deflection angle $(d)$ spectrum reconstructed from the same maps. Specifically, the lensing amplitude in CMB temperature and polarization spectra, $A_{\mathrm{L}}=1.22 \pm 0.10$, is in $2 \sigma$ tension with the amplitude of the $\mathrm{CMB}$ trispectrum reconstructed lensing deflection angle spectrum, $A_{\mathrm{L}}^{\phi \phi}=0.95 \pm 0.04$, while it is expected that in the base- $\Lambda$ CDM model, both of these quantities should be equal to unity.

The Planck Collaboration found that, compared with the base- $\Lambda \mathrm{CDM}$ model, the base- $\Lambda \mathrm{CDM}+A_{\mathrm{L}}$ model can reduce the logarithmic likelihood $\left(\Delta \chi^{2}=-6.1\right)$ and provide a better fit to the data sets with [1] $A_{\mathrm{L}}=1.28$ or a marginalized constraint $A_{\mathrm{L}}=1.22 \pm 0.10$ [2]. More importantly, they found that there is roughly equal preference for high $A_{\mathrm{L}}$ from intermediate and high multipoles (i.e., the PLIK likelihood; $\Delta \chi^{2}=-2.6$ ) and from the low- $\ell$ likelihood $\left(\Delta \chi^{2}=-3.1\right)$ with a further small change coming from the priors. This means that the base- $\Lambda \mathrm{CDM}+A_{\mathrm{L}}$ model can provide a better fit than the base- $\Lambda \mathrm{CDM}$ model against both TT and lowP data sets. However, the increase in $A_{\mathrm{L}}$ induces changes on the full sets of cosmological parameters as mentioned in Ref. [2]. For example, compared with the base- $\Lambda$ CDM fit, the scalar index $n_{s}$ is increased by $1 \%$, the primordial scalar spectrum amplitude $A_{s}$ is reduced by $4 \%$, and the effective amplitude of the TT spectrum $A_{s} e^{-2 \tau}$ is reduced by $1 \%$. Through the complicated relationship between parameters and their degeneracy, the reionization optical depth parameter $\tau$ falls to 0.060 , which is roughly in $2 \sigma$ tension with Planck-2013 temperature + WMAP low- $\ell$ polarization data results of $\tau=0.089_{-0.014}^{+0.012}$.

Inspired by these observations, in this paper, we investigate whether some modifications of gravity can relieve the tension between Planck CMB anisotropy spectra and CMB lensing results. To do so, we simulate a tension CMB data set that resembles the tension present in the Planck-2015 results, and we try to fit the resulting power spectra with different models to have a glimpse of the changes in the parameter that arise because of this tension.

\section{MOCK DATA AND FIDUCIAL PARAMETERS}

We analyze several modified gravity models against two sets of simulations of CMB spectra (TT, TE, EE) and CMB lensing spectra (dd and Td) with the fiducial cosmological parameter equal to the Planck-2015 data release best-fit values and Planck bluebook beam and noise specifications. The motivation for doing these simulations is mainly twofold. First, the Planck-2015 likelihood code and the corresponding spectrum data are not yet publicly available. Second, because the cosmological parameters are degenerate with each other in a complicated way, by using simulations, we can efficiently isolate and study the effects coming from different parameters and their combinations. 
TABLE I. Fiducial parameters of the mock data sets.

\begin{tabular}{lll}
\hline \hline $\mathrm{CP}$ & Mock-A & Mock-B \\
\hline $10^{9} A_{s}$ & 2.10745 & 2.14338 \\
$n_{s}$ & 0.97468 & 0.97156 \\
$\tau$ & 0.0611 & 0.0664 \\
$\Omega_{b} h^{2}$ & 0.022674 & 0.022379 \\
$\Omega_{c} h^{2}$ & 0.11639 & 0.11748 \\
$H_{0}$ & 69.02 & 68.39 \\
$A_{\mathrm{L}}$ & 1.28 & 1.02 \\
$\sum m_{\nu} / \mathrm{eV}$ & 0.06 & 0.06 \\
\hline \hline
\end{tabular}

${ }^{\mathrm{a}}$ Fiducial parameter values in Mock A data sets are the best-fit values of base- $\Lambda \mathrm{CDM}+A_{\mathrm{L}}$ to Planck-2015 TT + lowP data sets.

${ }^{b}$ Fiducial parameter values in Mock B data sets are the best-fit values of base- $\Lambda \mathrm{CDM}+A_{\mathrm{L}}$ to Planck-2015 TT + lowP + lensing data sets.

To construct our simulations, we use the cosmological parameters listed in Table I along with the CAMB code $[3,4]$ to produce the fiducial $\mathrm{CMB}$ temperature and $\mathrm{E}$-mode polarization power spectra. We feed these to the FuTURCMB [5] package to compute the noise power spectra for T, E-mode, and the lensing deflection angle based on the Hu-Okamoto [6] quadratic estimator. For further details about the FUTURCMB code, we refer the reader to [5] while for the construction of the spectrum likelihood, we refer to $[5,7,8]$. In this paper, we adopt the Planck bluebook [9] beam and noise parameters listed in Table II.

After the above operations, we build two mock data sets $\left(C_{\ell}^{T T}, C_{\ell}^{T E}, C_{\ell}^{E E}, C_{\ell}^{d d}\right.$, and $\left.C_{\ell}^{T d}\right)$, which we call mock-A and mock-B, whose fiducial cosmological parameter values (see Table I) are, respectively, the best-fit values of base- $\Lambda \mathrm{CDM}+A_{\mathrm{L}}$ to Planck-2015 TT + lowP and Planck-2015 TT + lowP + lensing data sets [10]. Since the mock-A data set, which mimics Planck-2015 TT + lowP, is generated from $A_{\mathrm{L}} \sim 1.3$, we can treat it as a realization of a non- $\Lambda \mathrm{CDM}$ universe; mock-B data, which mimic Planck-2015 TT + lowP + lensing, $A_{\mathrm{L}} \sim 1.0$, are closer to a realization of a $\Lambda \mathrm{CDM}$ universe.

Based on mock-A and mock-B data sets, we build a "tension" data set, called mock-C, by combining $\left(C_{\ell}^{T T}\right.$, $\left.C_{\ell}^{T E}, C_{\ell}^{E E}\right)$ from mock-A and $\left(C_{\ell}^{d d}\right.$ and $\left.C_{\ell}^{T d}\right)$ from mock-B. The resulting data set should mimic the tension present in Planck data while being free of additional complications

TABLE II. Planck bluebook instrumental specifications.

\begin{tabular}{lcccc}
\hline \hline Experiment & Frequency & $\theta_{\text {beam }}$ & $\sigma_{T}$ & $\sigma_{P}$ \\
\hline Planck: & 217 & 5.02 & 13.1 & 26.7 \\
& 143 & 7.30 & 6.0 & 11.4 \\
& 100 & 9.68 & 6.8 & 10.9 \\
\hline \hline
\end{tabular}

Frequencies in GHz. Beam size $\theta_{\text {beam }}$ is the FWHM in arcminutes. Sensitivities $\sigma_{T}$ and $\sigma_{P}$ are in $\mu K$ per FWHM beam. that arise in the real situation. Before the end of this section, we would like to emphasize that the strategy of simulating data has both strong and weak points. On one hand, this procedure allows us to single out specific effects that in the real data could be shaded by systematics or by noise, and this allows us to target precisely the physical phenomena under scrutiny. On the other hand, the simulated data set is not expected to reproduce precisely the results of the real one because there are a number of details about the likelihood construction that cannot possibly be mimicked. Our likelihood is built analytically at all multipoles without binning and assuming the same sky coverage in all frequency bands in temperature and polarization. In addition, we adopt all the polarization data into our numerical analysis, unlike in the lowP likelihood in which only the low- $\ell$ polarization data are used. The simulated lensing data are then reconstructed based on these power spectra, and the relative balance between the lensing and the T and $\mathrm{E}$ likelihoods results in assigning more weight to the $\mathrm{T}$ and $\mathrm{E}$ likelihood (because we include high- $\ell$ TE and EE data) with respect to real Planck (TT + lowP + lensing) results. This procedure for building the likelihood is not the same as those adopted by the Planck Collaboration, and the results on specific parameters reflect this point. We revisit this point in Sec. IV.

In the rest of the paper, we study several modified gravity models against mock- $\mathrm{A}$ and mock-C data sets to see whether the modified gravity models can or cannot reconcile the tension between CMB anisotropy data and CMB lensing data.

\section{MODIFIED GRAVITY IN THE EFFECTIVE FIELD THEORY FRAMEWORK}

In the one extra parameter extension of the base- $\Lambda \mathrm{CDM}$ model in the Planck-2013 [11] and Planck-2015 [2] results, the Planck Collaboration studied the case of varying the lensing amplitude parameter $A_{\mathrm{L}}$ in the CMB anisotropies, which was originally introduced in [12]. This phenomenological parameter is defined by $C_{\ell}^{\phi \phi} \rightarrow A_{\mathrm{L}} C_{\ell}^{\phi \phi}$, which simply rescales the lensing amplitude contribution to the CMB anisotropies. This parameter, however, only modulates the CMB anisotropy spectra, $C_{\ell}^{T T}, C_{\ell}^{T E}$, and $C_{\ell}^{E E}$, and rescales the lensing potential spectrum $C_{\ell}^{\phi \phi}$ but does not rescale the estimator of the lensing spectrum $\hat{A}_{\mathrm{L}}^{\phi \phi}$ which is computed from the CMB anisotropy trispectra [13,14]. For a review of $\mathrm{CMB}$ temperature and polarization lensing, we refer the reader to [15].

In the following, we elaborate on the relationship between this phenomenological parameter and modifications of gravity. We consider two models that are enclosed in the background part of the EFT formalism for cosmic acceleration $[16,17]$. Both can be derived from this action written in the unitary gauge and Jordan frame 


$$
\begin{aligned}
S= & \int d^{4} x \sqrt{-g}\left\{\frac{m_{0}^{2}}{2}[1+\Omega(\tau)] R+\Lambda(\tau)\right. \\
& \left.-a^{2} c(\tau) \delta g^{00}+\ldots\right\}+S_{m}\left[g_{\mu \nu}\right],
\end{aligned}
$$

where we have used conformal time, and $\Omega, \Lambda$, and $c$ are free functions of time which multiply all the operators that are consistent with time-dependent spatial diffeomorphism invariance and contribute to the background evolution. The ellipsis indicates operators which would affect only linear and nonlinear cosmological perturbations, while $S_{m}$ indicates the action for all matter fields: cold dark matter, baryons, massive and massless neutrinos, and photons.

The first modified gravity model that we use is $f(R)$; its mapping to the EFT framework was presented in [16], and we refer the reader to [18-21] for detailed discussions on the cosmology of these models.

The second model consists of taking a constant value for the conformal coupling $\Omega(a)=\Omega_{0}^{\mathrm{EFT}}$ and requiring the expansion history to be exactly that of the $\Lambda \mathrm{CDM}$ model. This requirement fixes, through the Friedmann equations, the time dependence of the operators $c$ and $\Lambda$.

We highlight here that the constant $\Omega$ model is not a simple redefinition of the gravitational constant. In fact, the requirement of having a $\Lambda \mathrm{CDM}$ background with a nonvanishing $\Omega$, which would change the expansion history, means that a scalar field is sourced in order to compensate this change. This scalar field interacts with the other matter fields and modify the behavior of cosmological perturbations and, consequently, the CMB power spectra and the growth of structure. For instance, it is easy to show that in the constant $\Omega$ model, $c(\tau)$, which is vanishing in general relativity, is nonzero and reads

$$
c=\frac{\Omega}{2}\left(\rho_{m}+P_{m}\right) .
$$

Another general remark we would like to make on the models that we consider here is that they display a radically different cosmology, as they correspond to two different behaviors of the perturbation's effective gravitational constant. Viable models, in the $f(R)$ case [19], correspond to an enhancement of the gravitational constant which in turn results in the amplification of the growth of structure that substantially enhances the lensing of the CMB.

In the second case, we consider two possibilities. If the constant $\Omega$ is positive, the model is characterized by a smaller effective gravitational constant resulting in a suppression of the growth and, consequently, a suppression of the CMB lensing. We call this case the $\Omega_{+}$model. If the constant $\Omega$ is negative, on the other hand, the model has an enhanced effective gravitational constant with a phenomenology similar to that of $f(R)$ models. In contrast to what happens to the $\Omega_{+}$case, which respects all the usual requirements of physical viability [22], this model is only classically stable. This means that perturbations around the Friedmann-Lemaitre-Robertson-Walker (FLRW) background are stable and well behaved but, for example, the sign of the scalar field kinetic term is wrong. We call the case in which the constant $\Omega$ can be greater and smaller than zero the $\Omega_{ \pm}$model.

To study the phenomenology of these three models, we use the EFTCAMB code [22-24] that allows us to compute cosmological observables for all the theories enclosed in the EFT formalism once a precise mapping or parametrization is given. The implementation details of the code and the equations that are solved can be seen in [25] with further comments on the models that we are considering.

\section{RESULTS AND DISCUSSIONS}

We first check the reliability of our simulations. As reported in the Table I, we use the fiducial $A_{\mathrm{L}}=1.28$ for the generation of the mock-A data set. After going through the Markov chain Monte-Carlo analysis [26], we get the marginalized constraint from the mock-A data set as

$$
A_{\mathrm{L}}=1.31 \pm 0.06(68 \% \text { C.L. }) .
$$

The consistency between the input and output values of our simulations can also be seen in the panels of Fig. 1. In addition, from Fig. 1(a) we can see that there is a relatively large positive correlation between $n_{s}$ and $A_{\mathrm{L}}$. This happens because a large value of $n_{s}$ enhances the high- $\ell$ multipoles, while an increase in $A_{\mathrm{L}}$ smears the peaks in the same multipole range. On the other hand, Fig. 1(b) shows that $\sigma_{8}$ and $A_{\mathrm{L}}$ are anticorrelated. An increase of $A_{\mathrm{L}}$ suppresses the growth inferred from the TT power spectrum; hence, we end up with a lower $\sigma_{8}$ value. Figure 1(c), at last, shows that no significant degeneracy arises between $A_{\mathrm{L}}$ and the reionization optical depth parameter $\tau$.

From all the panels of Fig. 1, we can see how different cosmological parameters react to the use of a tension data set. The shift of blue and red contours from mock-A and mock-C data sets shows the tension between them. In particular, in Fig. 1(a), we can see that the mock-C data set gives a value of $A_{\mathrm{L}} \sim 1.15$ that is in the middle of the mock-A $\left(A_{\mathrm{L}} \sim 1.3\right)$ and mock-B $\left(A_{\mathrm{L}} \sim 1.0\right)$ data sets. This happens because the mock-C likelihood is constructed from the equally weighted combination of the mock-A and mock-B ones, while in the real Planck results [2], the CMB lensing data dominate the constraint on $A_{\mathrm{L}}$.

From Figs. 1(b) and 1(c), we can instead see that the marginal probability distribution of $\sigma_{8}$ and $\tau$ does not change significantly as the tension in the data set is introduced. This happens because the constraint on these two quantities is dominated by the TT and EE power spectra. From the same panels, we notice that there is a slight bias in parameter estimation with respect to the real Planck data. This is due to differences in the construction of the likelihood that result in different weights being assigned to the temperature and polarization likelihood with respect 

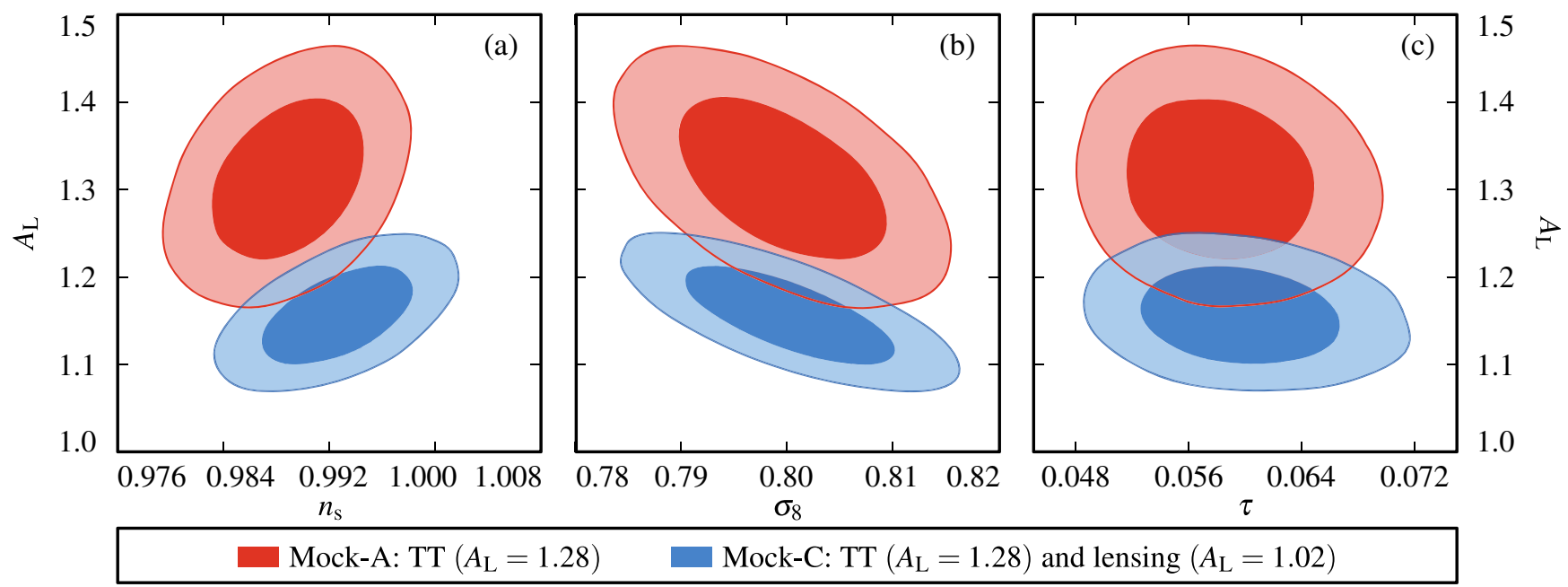

FIG. 1 (color online). (a) The marginalized joint likelihood for the lensing amplitude $A_{\mathrm{L}}$ and the scalar spectral index $n_{s}$, (b) the amplitude of the (linear) power spectrum on the scale of $8 h^{-1} \mathrm{Mpc}, \sigma_{8}$, and (c) the reionization optical depth $\tau$. In all three panels, different colors correspond to different combinations of mock data sets as shown in the legend. The darker and lighter shades correspond, respectively, to the $68 \%$ C.L. and the $95 \%$ C.L.

to the lensing one. As stressed at the beginning of this paper, this simulation procedure is targeted at mimicking the tension between the data sets rather than the data sets themselves and their specific constraints on cosmological parameters; therefore, this effect does not affect the following analysis of the results and conclusions.

After checking the consistency of our simulations, we move to their interpretation within the modified gravity models considered here.

First, we check whether these models can reproduce the input amplitude of lensing. To do so, we define an effective lensing amplitude as $A_{\mathrm{L}}^{\text {eff }}(\ell) \equiv C_{\ell}^{\phi \phi}(\mathrm{MG}) / C_{\ell}^{\phi \phi}(\mathrm{GR})$, and we plot it for several choices of the parameters defining our modified gravity models in Fig. 2. From both panels, we can see that the effective lensing amplitude is generally a function of scale so that the accuracy of using a scaleindependent approximation is limited by the accuracy of observations. That is, if observations are not precise enough, then the scale-independent approximation can work, while if the observations have enough sensitivity, we would bias parameter estimation by considering it to be scale independent. This point is more relevant as the experimental accuracy improves.

The $f(R)$ case, shown in Fig. 2(a), in particular, displays an $A_{\mathrm{L}}^{\text {eff }}$ that is monotonically increasing as a function of scale. At low multipoles, the agreement with a scaleindependent model is as good as a few percent, while in the high $-\ell$ range it is off by $40 \%$ depending on the present value of the Compton wavelength of the scalaron. The constant $\Omega_{+}$and $\Omega_{+}$models are shown in Fig. 2(b). Unlike the $f(R)$ case, $A_{\mathrm{L}}^{\text {eff }}(\ell)$ has a wide bump or dip around multipoles of a few tenths, while the scale dependence is somewhat weaker than the previous case. As expected, in the $\Omega_{+}$model the amplitude of lensing is decreased as a consequence of the suppression of growth, while the opposite behavior shows when $\Omega_{0}^{\mathrm{EFT}}$ is smaller than zero.

We fit these models to our mock-A and mock-C data sets, and we show the marginalized bound on parameters of interest in Fig. 3. From Fig. 3(a), we can see that there is a strong positive correlation between the present scalaron Compton wavelength parameter $B_{0}$ and $\sigma_{8}$. This effect is well known and corresponds to the fact that as the effective Newton constant is increased, the growth of matter perturbations is increased as well. When considering just mock-A, our results show that in order to mimic $A_{\mathrm{L}} \sim 1.3$, the marginalized constraint on $B_{0}$ has to point significantly toward large values of the scalaron Compton wavelength. The marginal bound is then $-1.15<\log _{10} B_{0}<-0.04$ at $95 \%$ C.L.

Due to the significant degeneracy between $B_{0}$ and $\sigma_{8}$, such values of $B_{0}$ leads to strong enhancement of the growth rate at relatively small scales making the $\sigma_{8}$ value too large so that the tension between Planck and LSS surveys, such as CFHTLenS [27,28], would become even worse. For this reason, when lensing is added in mock-C, the tension in $A_{\mathrm{L}}$, which for the lensing data set is smaller, pushes the posterior of $B_{0}$ toward smaller values, making it closer to GR. A similar effect was also observed for the Planck-2015 data set [29] and in the Planck-2013 data [30-33].

As expected, a similar result is found also in the $\Omega_{ \pm}$ model, as shown in Fig. 3(b). Here, the correlation between $\Omega_{0}^{\mathrm{EFT}}$ and $\sigma_{8}$ is negative due to the fact that negative values of $\Omega$ correspond to significant deviations from GR, and consequently, to stronger enhancement of CMB lensing. All the conclusions, previously discussed in the $f(R)$ case, apply also to this model with the relevant exception that the enhancement of the growth is not so dramatic as in $f(R)$. 
(a) effective lensing amplitude $f(R)$

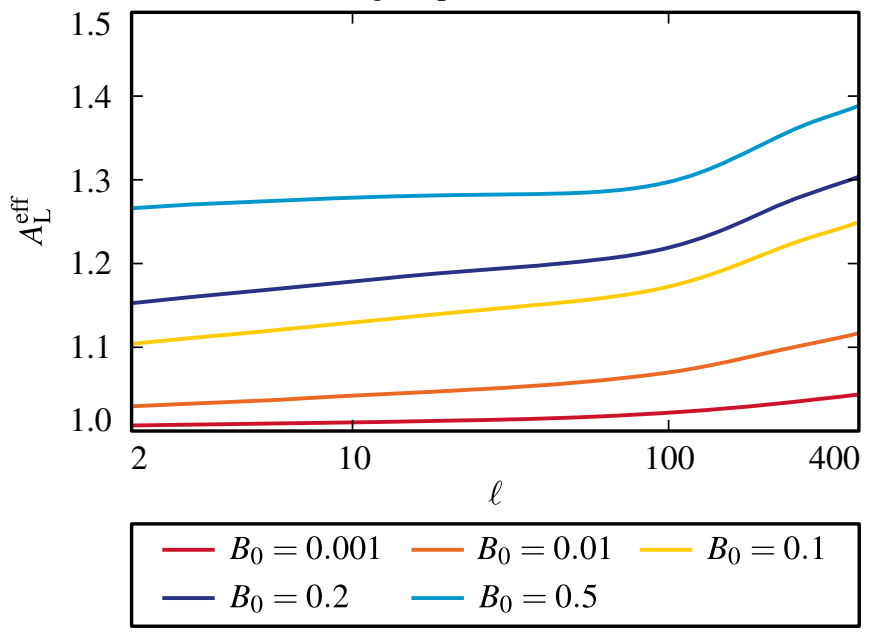

(b) effective lensing amplitude constant $\Omega$

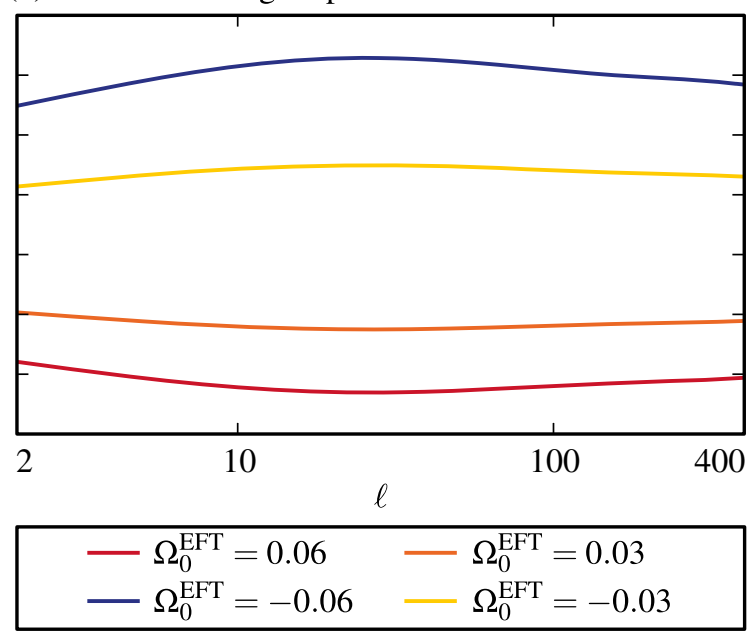

FIG. 2 (color online). The effective lensing amplitude $A_{\mathrm{L}}^{\mathrm{eff}}(\ell) \equiv C_{\ell}^{\phi \phi}(\mathrm{MG}) / C_{\ell}^{\phi \phi}(\mathrm{GR})$ as a function of scale in the modified gravity models considered in this paper. Different colors correspond to different values of the model parameters as shown in the legend.

The bound on $\sigma_{8}$ from the $A_{\mathrm{L}}$ fit, shown in Fig. 3 as a gray band, is in fact almost compatible with the one in the $\Omega_{ \pm}$ case. This is due to the fact that deviations from scale independence of $A_{\mathrm{L}}^{\mathrm{eff}}$, in this model, are weaker and a constant $\Omega$ is more efficient at mimicking a scaleindependent $A_{\mathrm{L}}$ than $f(R)$ models. Since a weaker effective Newton constant is disfavored by the Planck2015 CMB anisotropy data, the best-fit parameter in the $\Omega_{+}$model mimics those in the base- $\Lambda$ CDM model and the model result is compatible with GR. This conclusion is similar to the one found in [24,29,30].

Finally, in Table III, we list the best-fit $\chi^{2}$ for the one-parameter extensions of the base- $\Lambda \mathrm{CDM}$ model that we investigated in this paper. From there, we can see that the best-fit $\chi^{2}$ values in the base- $\Lambda \mathrm{CDM}+A_{\mathrm{L}}, f(R), \Omega_{ \pm}$ models are similar and are noticeably smaller than those from base- $\Lambda \mathrm{CDM}$ or $\Omega_{+}$models with $\Delta \chi^{2} \simeq-16$ from the mock-A and $\Delta \chi^{2} \simeq-11$ from the mock-C data sets. The best-fit $\chi^{2}$ in the $\Omega_{ \pm}$case is slightly better than the $f(R)$ case and closer to the $A_{\mathrm{L}}$ one because of the weaker scale dependence of $A_{\mathrm{L}}^{\mathrm{eff}}$.

In conclusion, Planck-2015 results revealed some interesting tensions between CMB temperature and polarization anisotropies and $\mathrm{CMB}$ lensing. These tensions add to the one reported by LSS surveys, like CFHTLenS, that seem to favor a smaller $\sigma_{8}$. If this is not due to some unaccounted (a) designer $\mathrm{f}(\mathrm{R})$

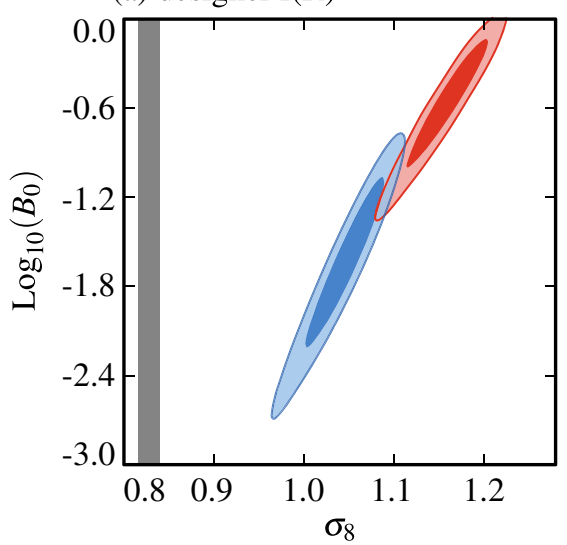

(b) constant $\Omega_{ \pm}$

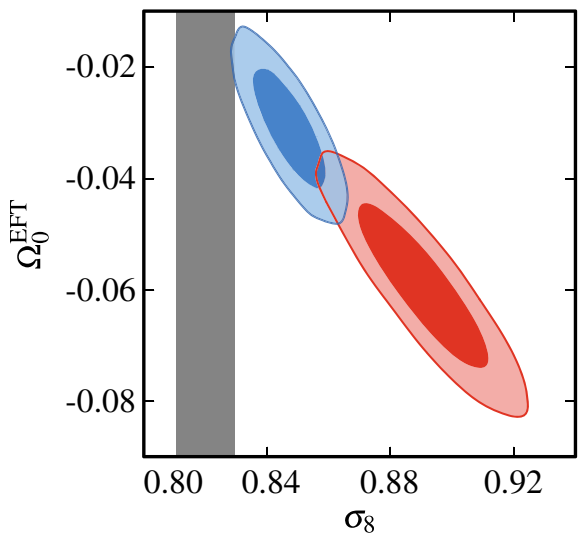

(c) constant $\Omega_{+}$

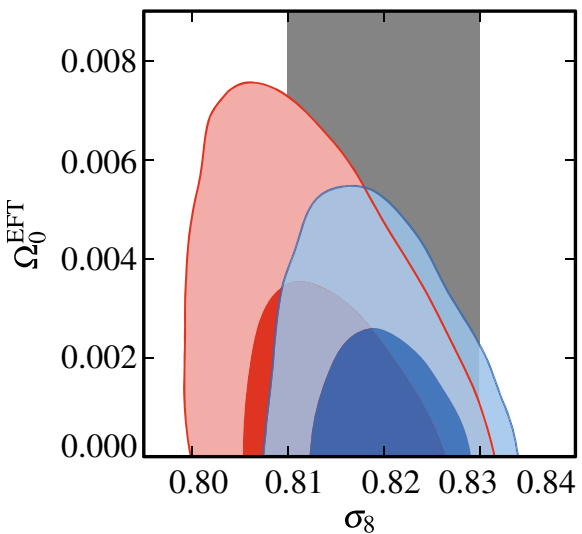

Mock-A: TT $\left(A_{\mathrm{L}}=1.28\right)$

Mock-C: TT $\left(A_{\mathrm{L}}=1.28\right)$ and lensing $\left(A_{\mathrm{L}}=1.02\right)$

FIG. 3 (color online). The marginalized joint likelihood for the amplitude of the (linear) power spectrum on the scale of $8 h^{-1} \mathrm{Mpc}, \sigma_{8}$, and the present value of $\log _{10} B_{0}$ panel (a), the present value of the conformal coupling $\Omega_{0}^{\mathrm{EFT}}$ in the case in which it is allowed to have positive and negative values panel (b) and in the case in which it is restricted to positive values panel (c). In all three panels, different colors correspond to different combination of mock data sets as shown in legend and the gray band is the marginalized $1 \sigma$ bound on $\sigma_{8}$ from the base- $\Lambda \mathrm{CDM}+A_{\mathrm{L}}$ model. The darker and lighter shades correspond, respectively, to the $68 \%$ C.L. and the 95\% C.L. 
TABLE III. The best-fit $\chi^{2}$.

\begin{tabular}{lcc}
\hline \hline$\chi_{\text {best-fit }}^{2}$ & Mock-A & Mock-C \\
\hline Base- $\Lambda$ CDM & 1967.373 & 3314.547 \\
Base- $\Lambda$ CDM $+A_{\mathrm{L}}$ & 1951.122 & 3303.599 \\
$f(R)$ & 1952.325 & 3305.251 \\
$\Omega_{+}$ & 1967.604 & 3314.676 \\
$\Omega_{ \pm}$ & 1951.109 & 3304.507 \\
\hline \hline
\end{tabular}

for systematic effects, this might be an indication of exotic physics beyond the base- $\Lambda \mathrm{CDM}$ model and, as such, should be investigated in detail.

In this paper, using a simulation of the Planck-2015 data set, we tried to reconcile this tension with some modified gravity models. In particular, we studied whether this tension can be mitigated by $f(R)$ models or models characterized by a constant conformal coupling between gravitational and matter perturbations. We found that the $f(R)$ and the $\Omega_{ \pm}$models can mimic the role of $A_{\mathrm{L}}$ even if, generally, the amplitude of lensing, with respect to the GR case, is scale dependent. In order to provide a good fit to the Planck-like CMB anisotropy spectra, however, these models predict an enhancement of the growth on smaller scales that make the tension in $\sigma_{8}$ even worse. From these results, we can conclude that the tension between the growth of matter perturbations assessed from $\mathrm{CMB}$ power spectra, CMB lensing, and LSS surveys can be mimicked by modified gravity models but is hardly relieved by simple models beyond the standard $\Lambda \mathrm{CDM}$ one. This in turn suggests that one should investigate more complicated models, possibly with different time dependencies, to allow for different regimes of growth at the times at which each of these observations is more sensitive.

\section{ACKNOWLEDGMENTS}

We thank Carlo Baccigalupi, Julien Lesgourgues, Matteo Martinelli, Sabino Matarrese, Laurence Perotto, Alessandra Silvestri, Wessel Valkenburg, and Matteo Viel for helpful correspondences and discussions. B. H. is supported by the Dutch Foundation for Fundamental Research on Matter (FOM). M. R. acknowledges partial support from the INFN-INDARK initiative. M. R. thanks the Instituut Lorentz (Leiden University) for hospitality while this work was being completed.
[1] From the PLA-PR2-2015 official chains BASE-ALENSPLIKHM-TT-LOwTEB at http://pla.esac.esa.int/pla/.

[2] P. A. R. Ade et al. (Planck Collaboration), arXiv: 1502.01589.

[3] A. Lewis, A. Challinor, and A. Lasenby, Astrophys. J. 538, 473 (2000).

[4] http://camb.info.

[5] L. Perotto, J. Lesgourgues, S. Hannestad, H. Tu, and Y. Y. Y. Wong, J. Cosmol. Astropart. Phys. 10 (2006) 013.http://lpsc .in2p3.fr/perotto/.

[6] W. Hu and T. Okamoto, Astrophys. J. 574, 566 (2002).

[7] M. Tegmark, A. Taylor, and A. Heavens, Astrophys. J. 480, 22 (1997).

[8] A. Lewis, Phys. Rev. D 71, 083008 (2005).

[9] J. Tauber et al. (Planck Collaboration), arXiv:astro-ph/ 0604069 .

[10] The best-fit values are read from the PLA-PR2-2015 official chains BASE-ALENS-PLIKHM-TT-LOWTEB and BASEALENS-PLIKHM-TT-LOWTEB-LENSING at http://pla.esac .esa.int/pla/.

[11] P. A. R. Ade et al. (Planck Collaboration), Astron. Astrophys. 571, A16 (2014).

[12] E. Calabrese, A. Slosar, A. Melchiorri, G. F. Smoot, and O. Zahn, Phys. Rev. D 77, 123531 (2008).

[13] P. A. R. Ade et al. (Planck Collaboration), arXiv: 1502.01591.

[14] P. A. R. Ade et al. (Planck Collaboration), Astron. Astrophys. 571, A17 (2014).

[15] A. Lewis and A. Challinor, Phys. Rep. 429, 1 (2006).
[16] G. Gubitosi, F. Piazza, and F. Vernizzi, J. Cosmol. Astropart. Phys. 02 (2013) 032.

[17] J. K. Bloomfield, É. É. Flanagan, M. Park, and S. Watson, J. Cosmol. Astropart. Phys. 08 (2013) 010.

[18] Y. S. Song, W. Hu, and I. Sawicki, Phys. Rev. D 75, 044004 (2007).

[19] L. Pogosian and A. Silvestri, Phys. Rev. D 77, 023503 (2008); , 81, 049901(E) (2010).

[20] R. Bean, D. Bernat, L. Pogosian, A. Silvestri, and M. Trodden, Phys. Rev. D 75, 064020 (2007).

[21] A. De Felice and S. Tsujikawa, Living Rev. Relativity 13, 3 (2010).

[22] B. Hu, M. Raveri, N. Frusciante, and A. Silvestri, Phys. Rev. D 89, 103530 (2014).

[23] Publicly available at: http://wwwhome.lorentz.leidenuniv nl/ hu/codes/.

[24] M. Raveri, B. Hu, N. Frusciante, and A. Silvestri, Phys. Rev. D 90, 043513 (2014).

[25] B. Hu, M. Raveri, N. Frusciante, and A. Silvestri, arXiv: 1405.3590.

[26] A. Lewis and S. Bridle, Phys. Rev. D 66, 103511 (2002).

[27] C. Heymans, L. Van Waerbeke, L. Miller, T. Erben, H. Hildebrandt, H. Hoekstra, T. D. Kitching, Y. Mellier et al., Mon. Not. R. Astron. Soc. 427, 146 (2012).

[28] T. Erben, H. Hildebrandt, L. Miller, L. van Waerbeke, C. Heymans, H. Hoekstra, T. D. Kitching, Y. Mellier et al., Mon. Not. R. Astron. Soc. 433, 2545 (2013).

[29] P. A. R. Ade et al. (Planck Collaboration), arXiv: 1502.01590 
[30] B. Hu, M. Raveri, A. Silvestri, and N. Frusciante, Phys. Rev. D 91, 063524 (2015).

[31] J. Dossett, B. Hu, and D. Parkinson, J. Cosmol. Astropart. Phys. 03 (2014) 046.
[32] B. Hu, M. Liguori, N. Bartolo, and S. Matarrese, Phys. Rev. D 88, 123514 (2013).

[33] A. Marchini and V. Salvatelli, Phys. Rev. D 88, 027502 (2013). 